\title{
EXPORT: Spectral classification and projected rotational velocities of Vega-type and pre-main sequence stars ${ }^{\star, \star \star}$
}

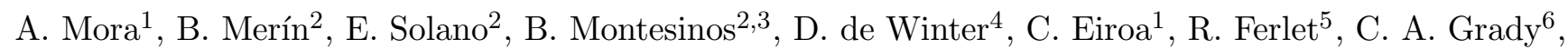
J. K. Davies ${ }^{7}$, L. F. Miranda ${ }^{3}$, R. D. Oudmaijer ${ }^{8}$, J. Palacios ${ }^{1}$, A. Quirrenbach ${ }^{9}$, A. W. Harris ${ }^{10}$, H. Rauer ${ }^{10}$, A. Cameron ${ }^{11}$, H. J. Deeg ${ }^{3}$, F. Garzón ${ }^{12}$, A. Penny ${ }^{13}$, J. Schneider ${ }^{14}$, Y. Tsapras ${ }^{11}$, and P. R. Wesselius ${ }^{15}$

1 Departamento de Física Teórica, Módulo C-XI, Facultad de Ciencias, Universidad Autónoma de Madrid, 28049 Cantoblanco, Madrid, Spain

2 Laboratorio de Astrofísica Espacial y Física Fundamental (LAEFF), Apartado de Correos 50727, 28080 Madrid, Spain

3 Instituto de Astrofísica de Andalucía-CSIC, Apartado de Correos 3004, 18080 Granada, Spain

4 TNO/TPD-Space Instrumentation, Stieltjesweg 1, PO Box 155, 2600 AD Delft, The Netherlands

${ }^{5}$ CNRS, Institut d'Astrophysique de Paris, 98bis Bld. Arago, 75014 Paris, France

6 NOAO/STIS, Goddard Space Flight Center, Code 681, NASA/GSFC, Greenbelt, MD 20771, USA

7 Joint Astronomy Centre, 660 N. A'ohoku Place, Hilo, Hawaii 96720, USA

8 Department of Physics and Astronomy, University of Leeds, Leeds LS2 9JT, UK

9 Department of Physics, Center for Astrophysics and Space Sciences, University of California San Diego, Mail Code 0424, La Jolla, CA 92093-0424, USA

10 DLR Department of Planetary Exploration, Rutherfordstrasse 2, 12489 Berlin, Germany

11 Department of Physics and Astronomy, University of St. Andrews, North Haugh, St. Andrews KY16 9SS, Scotland, UK

12 Instituto de Astrofísica de Canarias, c/Via Láctea s/n, 38200 La Laguna, Tenerife, Spain

13 Rutherford Appleton Laboratory, Chilton, Didcot, Oxfordshire OX11 0QX, UK

14 Observatoire de Paris, place Jules Janssen, 92195 Meudon, France

15 SRON, Universiteitscomplex "Zernike", Landleven 12, PO Box 800, 9700 AV Groningen, The Netherlands

Received 2 April 2001 / Accepted 30 July 2001

\begin{abstract}
In this paper we present the first comprehensive results extracted from the spectroscopic campaigns carried out by the EXPORT (EXoPlanetary Observational Research Team) consortium. During 1998-1999, EXPORT carried out an intensive observational effort in the framework of the origin and evolution of protoplanetary systems in order to obtain clues on the evolutionary path from the early stages of the pre-main sequence to stars with planets already formed. The spectral types of 70 stars, and the projected rotational velocities, $v \sin i$, of 45 stars, mainly Vega-type and pre-main sequence, have been determined from intermediate- and high-resolution spectroscopy, respectively. The first part of the work is of fundamental importance in order to accurately place the stars in the HR diagram and determine the evolutionary sequences; the second part provides information on the kinematics and dynamics of the stars and the evolution of their angular momentum. The advantage of using the same observational configuration and methodology for all the stars is the homogeneity of the set of parameters obtained. Results from previous work are revised, leading in some cases to completely new determinations of spectral types and projected rotational velocities; for some stars no previous studies were available.
\end{abstract}

Key words. stars: fundamental parameters - stars: pre-main sequence - stars: rotation

Send offprint requests to: A. Mora,

e-mail: alcione.mora@uam.es

* Tables 1 and 2 are only, and Table 6 also, available in electronic form at the CDS via anonymous ftp to cdsarc.u-strasbg.fr(130.79.128.5) or via http://cdsweb.u-strasbg.fr/cgi-bin/qcat?J/A+A/378/116

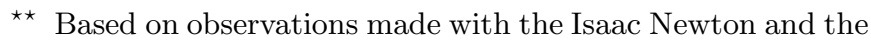
William Herschel telescopes operated on the island of La Palma by the Isaac Newton Group in the Spanish Observatorio del Roque de los Muchachos of the Instituto de Astrofísica de Canarias. 


\section{Introduction}

In the last few years interest in the formation and evolution of planetary systems has been boosted by the discovery of the first extrasolar planet orbiting around 51 Peg (Mayor \& Queloz 1995). Since then, many planets have been detected and the number of new objects increases steadily.

In addition, the presence of infrared excesses in the spectral distributions of Vega, $\beta$ Pic and other mainsequence stars is now commonly accepted to be caused by dust particles in disks around these objects (Aumann et al. 1984; Smith \& Terrile 1984). Transient spectroscopic events in the ultraviolet and optical lines of $\beta \mathrm{Pic}$, and asymmetries in the shape of its disk, have been interpreted in terms of infalling cometary bodies and even by the presence of a planet around this star (Lecavelier des Étangs 2000).

There is mounting evidence of a clear evolutionary chain leading from pre-main sequence stars (PMS hereafter) with infrared excesses in their spectra, i.e. circumstellar envelopes or disks, to stars with planets already formed (see e.g. Mannings et al. (ed.) 2000, part II). Therefore, it is an attractive perspective to study the evolutionary stellar path from the very early stages of pre-main sequence evolution, where even a population of stars might be $\beta$ Pic progenitors (Grady et al. 2000), to the main sequence, where, according to the results of the radial velocity surveys, a fraction of the solar-type stars may have massive planets. This was the philosophy behind the international observing time at the observatories in the Canary Islands allocated in 1998 to the EXPORT (EXoPlanetary Observational Research Team) collaboration. A large sample of PMS and main-sequence stars, was observed simultaneously with four telescopes, collecting high and intermediate resolution spectroscopy, optical and near-infrared photometry and polarimetry (Eiroa et al. 2000; Eiroa et al. 2001; Oudmaijer et al. 2001).

One of the goals of the proposed research was to obtain detailed information on the evolution of protoplanetary disks, a prerequisite of which is the determination of physical parameters of the stars in order to accurately place them in a HR diagram and establish evolutionary sequences for objects with different masses. Therefore, the rational underlying the choice of the sample was to select a fairly large group of stars of different classes, namely Vega-type, Herbig Ae/Be, classical and early $\mathrm{T}$ Tauri, most of them archetypal and representative of their group, although obviously, not unique. The environments where these stars are placed are not related, because we wished to observe objects with different ages. There is a balance between cool and hot objects: Among the 70 stars observed in intermediate resolution, 26 are low-mass - spectral type F or later - objects, so they represent $37 \%$ of the whole sample.

In this paper we present the spectroscopic data of the stars in the sample observed. From these, we have determined the spectral types and the projected rotational velocities. The first is an essential step since a homogeneous classification of all the stars, using the same observational configuration, method and standards, is the most reliable way for obtaining a coherent set of parameters for the whole sample; in addition, some of the stars observed had poorly accurate or incomplete classifications. The determination of rotational velocities will have implications on the dynamics and kinematics of the potential disks and therefore on the evolution of the protoplanetary systems around the stars.

The layout of the paper is as follows. In Sect. 2 we give some notes on the nomenclature used in the paper. In Sect. 3 we describe the observations and the reduction procedures. In Sect. 4, we give details on the tools used and processes followed to carry out the spectral classification; the results of this classification are given and discussed. Section 5 is devoted to show the method used to determine the projected angular velocities and the results obtained. The final remarks are given in Sect. 6 .

\section{Some notes on nomenclature}

Through this work we will use some terminology that is worth clarifying. The PMS can be divided in a first approximation into $\mathrm{T}$ Tauri and Herbig Ae/Be stars. The former are the precursors of solar-type stars, with spectral types later than F0, whereas the latter are precursors of stars with an intermediate range of masses and have spectral types earlier than F0. Both of them show emission lines in their spectra. In their turn, the $\mathrm{T}$ Tauri stars can be grouped into classical T Tauri stars (CTTs), with spectral types G2 and later, and early-type T Tauri stars (ETTs), with spectral types between F0 and G2.

The so-called Post $\mathrm{T}$ Tauri stars are older than the CTTs but they have not yet reached the main sequence. From the infrared emission discovered around some Post $\mathrm{T}$ Tauri stars (Ray et al. 1995) and the radio emission, it has been inferred that the amount of dust in the disks around these stars is one or two orders of magnitude less than that in the disks around CTTs and Herbig Ae/Be. The specific PTT subset in the EXPORT sample was taken from Lindroos' catalogue (Lindroos 1986) of visual binary systems composed of a primary main-sequence star with an early spectral type and a late-type secondary thought to be in the PTT phase.

In the main sequence we distinguish between the Vegatype stars and the A-shell stars. The Vega-type stars are named after their prototype, $\alpha$ Lyr, and are main-sequence stars with dusty disks - i.e. showing infrared excess; $\beta$ Pic is a typical Vega-type star. The A-shell stars are A stars in fast rotation showing non-photospheric absorption components in Ca II and in the Balmer lines, especially in H $\alpha$. Grady et al. (1996) detected, from ultraviolet spectra, material falling onto this kind of star, showing that the accretion phenomenon is not exclusive of the Vega-type stars. Finally, by MS we denote normal main-sequence stars without any infrared excess in their spectra. 


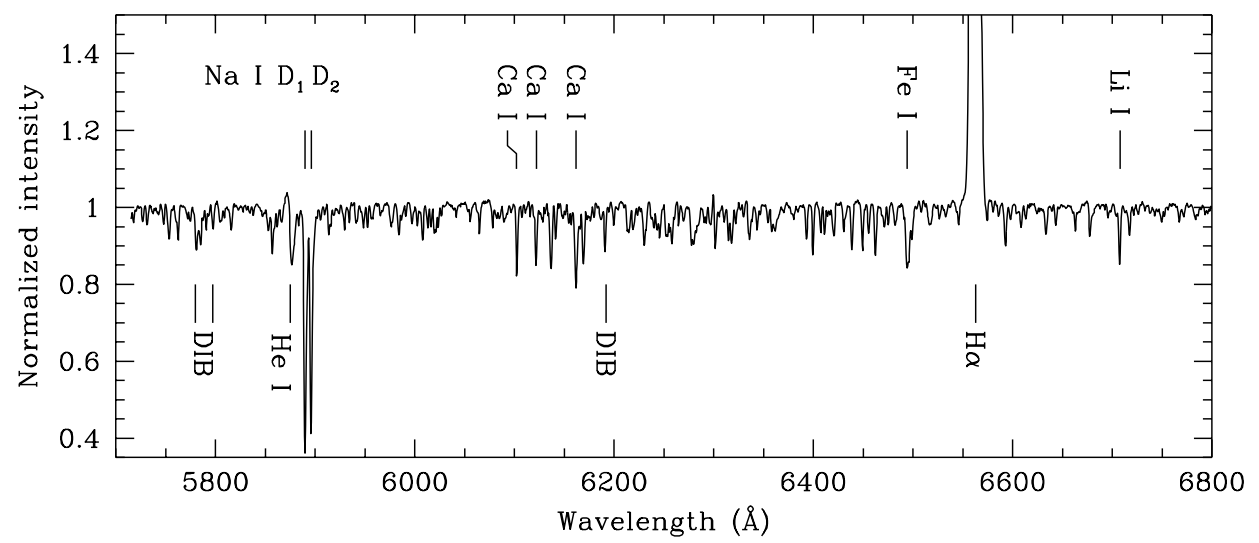

Fig. 1. The normalized average INT spectrum of BM And. Above the spectrum we give the identification of some prominent lines: Na I 5890 and $5896 \AA$, Ca I 6102, 6122 and $6162 \AA$, Fe I $6495 \AA$ and Li I $6708 \AA$. Below the spectrum we show some other lines formed, at least partially, in the interstellar or circumstellar medium: DIB's at 5780, 5797 and $6192 \AA$, He I $5875 \AA$ and H $\alpha 6563 \AA$. The Na I lines may also have an interstellar component.

\section{The observations and reduction procedures}

Intermediate resolution spectra obtained with the $2.5 \mathrm{~m}$ Isaac Newton Telescope (INT) and high resolution spectra obtained with the $4.2 \mathrm{~m}$ William Herschel Telescope (WHT), both at the Observatorio del Roque de los Muchachos (La Palma), were used for the spectral classification and for the determination of the rotational velocities, respectively. In the following two subsections we describe the details of each set of observations and some noteworthy aspects of the reduction procedures. A list of the stars observed with each telescope, dates and number of spectra, can be found in Tables 1 and 2 . The reduced observations can be requested to EXPORT under certain conditions; please contact Carlos Eiroa (carlos.eiroa@uam.es) for details.

\subsection{Intermediate resolution spectroscopy}

The observations were taken with the INT equipped with the Intermediate Dispersion Spectrograph (IDS) during four observing campaings: 14-17 May, 28-31 July, 23-27 October 1998 and 28-31 January 1999. This distribution of dates allowed us to do short-term (hours) and medium- to long-term (days, months) spectroscopic monitoring of the stars.

Two different instrumental setups were used. An EEV CCD was used in the May 1998 run, the spectral range covered from 5854 to $6728 \AA$, with a resolving power of $\sim 4500$. An upgraded EEV CCD was used during the other three observing runs. The wavelength coverage was 5712 to $6812 \AA$, with a resolving power of $\sim 6600$. The slit width was always set to 1.0 arcsec projected on the sky.

During the four runs, 70 target stars were observed, and a total of 491 individual spectra collected; 28 spectroscopic standards were also observed (see Sect. 4). The standard extraction and reduction procedures were used and the spectra were normalized to their continua in order to carry out the spectral classification by comparing the relative intensity of spectral features of the target stars with the same features in a grid of standard stars (see Sect. 4). In Fig. 1 we show an example of a typical INT spectrum for one of the stars in the sample, the $\mathrm{T}$ Tauri star BM And with some line identifications.

\subsection{High resolution spectroscopy}

The high resolution spectra were obtained with the WHT equipped with the Utrecht Echelle Spectrograph (UES). The observing runs coincided with those above-mentioned for the INT, but two nights in the May run and two in the October run were used for systematic monitoring of $\tau$ Boo and 51 Peg (Harris et al. 2000).

UES was set to provide a wavelength coverage between 3800 and $5900 \AA$. The spectra were dispersed into 59 echelle orders with a resolving power of 49000 . The slit width was set to 1.15 arcsec projected on the sky. A total of 47 stars were observed and 189 spectra obtained.

The echelle reduction procedure was divided into seven steps: order detection, background subtraction, filtering of cosmic rays, flat-field correction, spectrum extraction, wavelength calibration and blaze correction. The MIDAS echelle context was used to perform the first six stages, while the IRAF continuum fitter was selected to flatten the orders of the spectra.

The detection of the orders was performed using a bright standard star and the Hough transform method. The background was modelled by a two-dimensional cubic spline smooth surface, obtained by sampling the interorder space. Cosmic rays were detected and eliminated by comparing every single spatial profile at every order with a local average profile. The flat-field correction frames were obtained with a tungsten lamp. An optimal extraction was performed defining a different numerical slit for each target star, which gathered the light within a $3 \sigma$ Gaussian interval around the centre of each order.

The wavelength calibration was done using $\mathrm{Th}-\mathrm{Ar}$ and Fe-Ar arc lamp exposures. The calibration errors are typically about $20 \mathrm{~m} \AA(\sim 0.4$ pixels) and lower than the spectral resolution $(\sim 100 \mathrm{~m} \AA)$. The lower sensitivity at the order edges typical of echelle spectra (blaze effect) was 


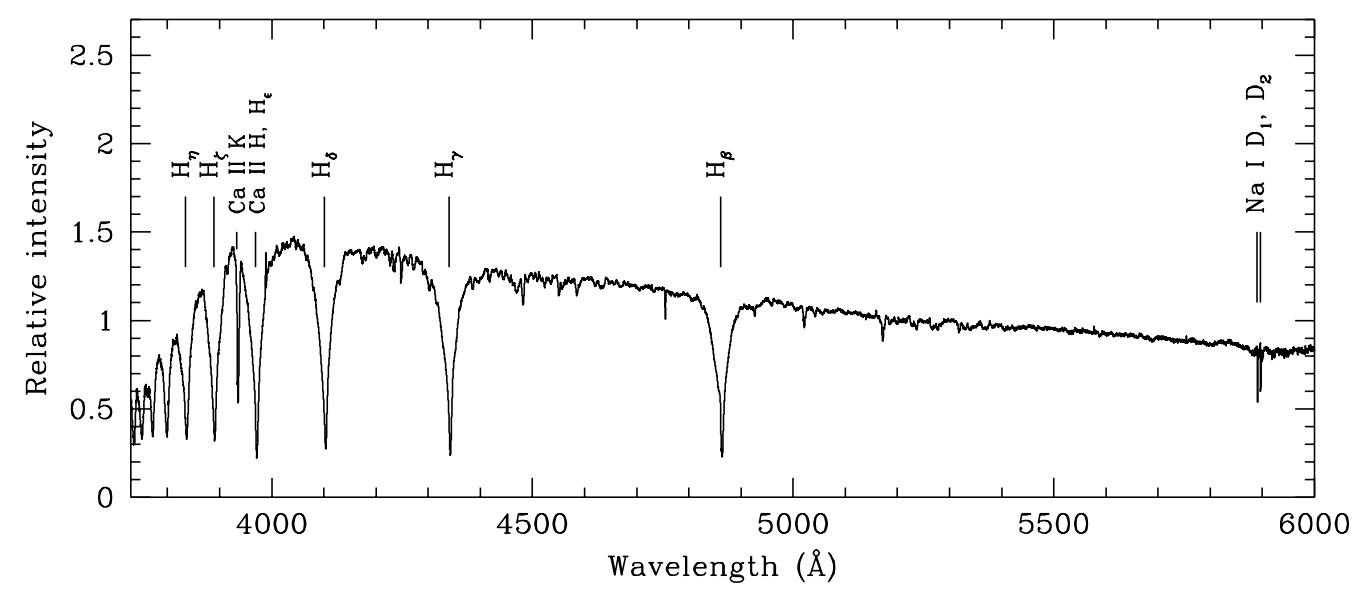

Fig. 2. The WHT merged spectrum of UX Ori. Some stellar lines affected by circumstellar material are shown: H $\eta 3835 \AA$,

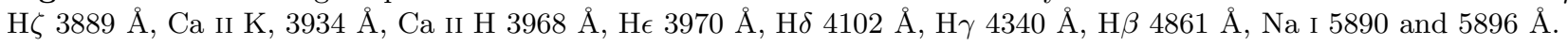

corrected by fitting the continuum of the standard stars observed. A blaze function was derived for each order. In Fig. 2 a WHT spectrum of the star UX Ori, including some line identifications, is shown.

\section{The spectral classification}

The determination of the precise spectral type of stars with circumstellar material is extremely useful. The immediate result of the classification is a derivation of the effective temperature that allows us to fix the position of the star in the $T_{\text {eff }}$ axis of a luminosity-effective temperature HR diagram. In addition, the comparison of the target spectra with spectra of standard stars may reveal the presence of non-standard features, which provide valuable information on the physical conditions of the photospheres and the surrounding medium.

\subsection{Methodology}

The spectral classification was done mainly by comparing the INT spectra of the target stars with spectra of standard stars obtained with the same or a very similar instrumental configuration.

Two sets of standard stars were used for the spectral classification: 28 standards observed with the INT during the four EXPORT campaigns; and a library of spectra of 116 late-type (F to M) field stars (Montes et al. 1997).

The instrumental configuration used to observe the standards with the INT was the same as the one used for the target stars. The spectra contained in the library compiled by Montes et al. (1997) were observed with different telescopes - the INT was one of them - and cover different spectral intervals with different resolutions. The closest configuration to those used during the EXPORT runs is the one labelled with number " 8 " in the paper by Montes et al.; it covers the range 5626 to $7643 \AA$, therefore, it overlaps the spectral interval of the target stars completely, although the spectral resolution is lower $(1.58 \AA$ per pixel).

The first step in the classification procedure was a visual inspection of the average spectrum and each one of the individual target spectra. This analysis allowed us to identify significant changes in the spectral type, which lines are variable, the presence of permitted and forbidden lines appearing in emission, and lines caused by interstellar absorption.

The spectra of the standard stars were broadened with the rotation profile corresponding to the target star, and then a comparison among them was carried out. When available, values of $v \sin i$ obtained by us (see Sect. 5) were used; otherwise, published values were taken at this point. This usually provided quite an exact determination of the spectral type. In some cases the classification was assigned by bracketing the spectrum of the target star between those of two standards with slightly different spectral types, taking the mean as the correct result. In Fig. 3 we can see an example of the efficiency of this step. In the upper panel the spectrum of the target star HD 199143 (solid line) is shown along with the spectrum of the slow-rotator $\left(v \sin i<5 \mathrm{~km} \mathrm{~s}^{-1}\right)$ standard star HR 4606 (F6 V) (dotted line); in the lower panel, the spectrum of the standard has been broadened with a rotation profile $v \sin i=155 \mathrm{~km} \mathrm{~s}^{-1}$. The match with the spectrum of the problem star is remarkable.

The presence of veiling in some of the stars in the sample, mainly CTTs, poses an additional difficulty in the determination of spectral types. Since the effect of veiling is a global weakening, or even the disappearance of the absorption features in the spectral region affected, the comparison of a spectrum affected by veiling with the grid of spectra of normal stars would lead to the derivation of an earlier spectral type to the target star instead of the correct one, therefore this effect must be treated properly. We illustrate this problem with a typical case, that of DR Tau, in Sect. 4.4.

Some additional tools may be applied for confirming the spectral classification of these stars. One of them is the use of ultraviolet spectra. For those objects observed with IUE (International Ultraviolet Explorer) ${ }^{1}$, a comparison with synthetic energy distributions computed using the

\footnotetext{
1 http://ines.vilspa.esa.es
} 


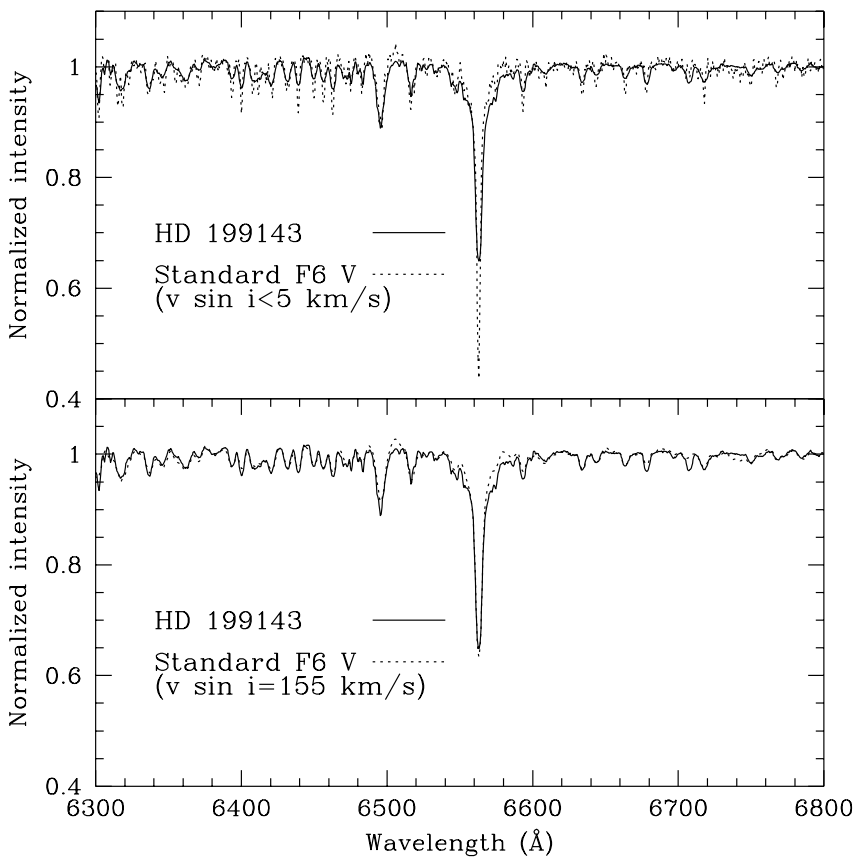

Fig. 3. Upper panel: the spectrum of HD 199143 (solid line) and the spectrum of a slow-rotator standard star HR 4606 (F6 V) (dotted line). Lower panel: the spectrum of the standard has been broadened with $v \sin i=155 \mathrm{~km} \mathrm{~s}^{-1}$.

ATLAS9 code (Kurucz 1993) was done. The ultraviolet spectra provided by $I U E$ are calibrated in absolute flux, so a direct comparison, after correcting for reddening, with the synthetic models was feasible.

The IUE spectra themselves have been used to estimate $E(B-V)$, taking advantage of the wide feature in absorption around $2200 \AA$ caused by the interstellar medium. The process consisted of correcting the spectra for reddening with different values of $E(B-V)$ until the absorption feature disappeared; at this point that particular value of $E(B-V)$ was assigned to the star. The Galactic extinction law by Howarth (1983) was used. This method is applicable only when there is a substantial amount of flux in the continuum around $2200 \AA$, i.e. it is only suitable for stars hotter than F0. Otherwise, published values of the colour excesses were used. In Table 3 we give the values of the colour excesses and their uncertainties determined in this way; in the last column values of $E(B-V)$ computed as $(B-V)_{\text {target star }}-(B-V)_{\text {st }}$ are given, being $(B-V)_{\text {st }}$ the colour index of a standard with the same spectral type as the target star. An uncertainty of 0.05 in the colour index leads to an error in the classification of three subtypes, so the more dubious confirmation of the spectral type following this method would be for VX Cas.

In 27 stars, this confirmed the spectral type obtained from the optical spectra. The use of IUE spectra is particularly powerful for Vega-type and A-shell stars since the spectral region covered $(\sim 1150-3350 \AA)$ is not affected by excesses or veiling. In the remaining cases, an accurate determination of the spectral type in the ultraviolet requires the knowledge of these peculiar features (Catala \& Bertout 1990; Valenti et al. 2000) and also of the potential
Table 3. $E(B-V)$ values from IUE spectra.

\begin{tabular}{llc}
\hline Star & $E(B-V)_{I U E}$ & $E(B-V)_{\text {target-st }}$ \\
\hline HD 58647 & $0.16 \pm 0.02$ & 0.16 \\
HD 179218 & $0.10 \pm 0.05$ & 0.08 \\
VX Cas & $0.30 \pm 0.10$ & 0.26 \\
17 Sex & $0.07 \pm 0.02$ & 0.02 \\
WW Vul & $0.20 \pm 0.05$ & 0.30 \\
\hline
\end{tabular}

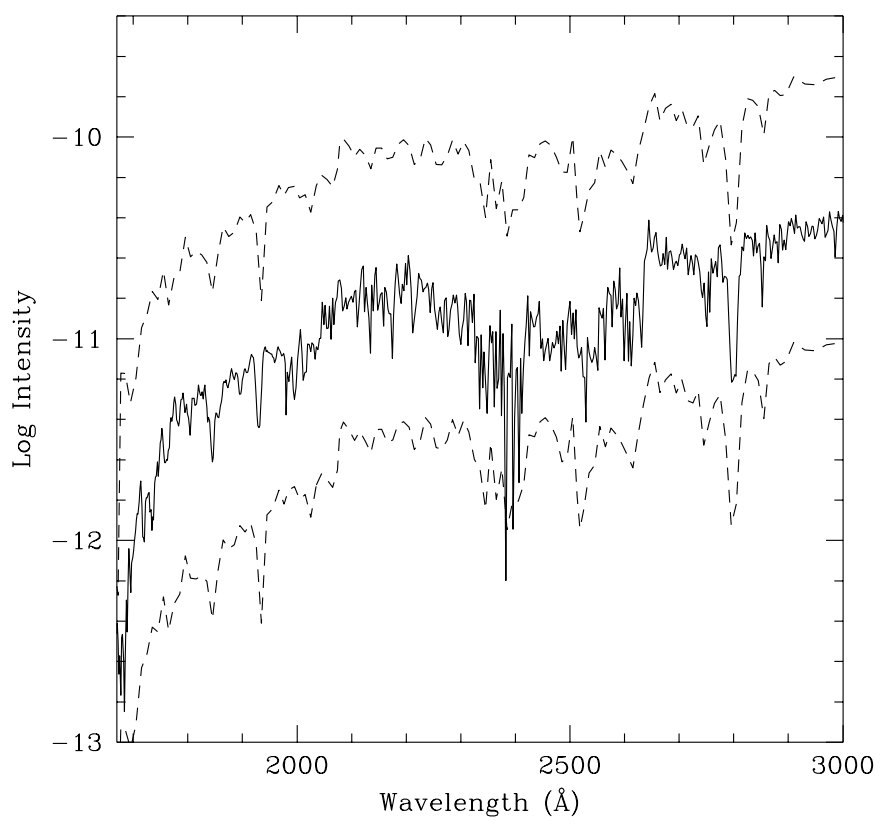

Fig. 4. An example of fitting of synthetic energy distributions (dashed lines) to the IUE spectrum of a Vega type star, HD 109085 (solid line). The two models correspond to spectral types F1 V (top) and F3 V (bottom). The original models were normalized to the target spectrum and shifted for clarity. The spectral type derived for HD 109085 from optical spectra is $\mathrm{F} 2 \mathrm{~V}$.

variability of the object in this range. In any case, the fact must be taken into account that the $I U E$ spectra are not simultaneous with the EXPORT observations. Different determinations of the spectral type from optical and ultraviolet observations might be attributable to long-term intrinsic variability of the stars.

In Fig. 4 we can see an example of a fit, in the ultraviolet range, of two synthetic Kurucz's models to the spectrum of HD 109085, a Vega-type star with a spectral type $\mathrm{F} 2 \mathrm{~V}$ as determined from the comparison with optical spectra of standards. We show the ordinates in logarithmic units. The two models (dashed lines) correspond to spectral types F1 V (top) and F3 V (bottom) and were computed with the solar abundance. The synthetic energy distributions were normalized to the target spectrum (solid line) and shifted 0.7 dex up and down for clarity. The similarity of the synthetic spectra with the energy distribution of the target star is remarkable.

The derivation of the luminosity class was also done by comparing the target spectra with those of standards with different luminosity classes. A consistent check of this 
Table 4. Useful lines to classify cool stars.

\begin{tabular}{l|l}
\hline Line identification & Spectral types \\
\hline Ca I $6102 \AA$ & \\
Ca I $6122 \AA$ & K0 V-K5 V \\
Fe I $6129 \AA$ & \\
\hline Fe I $6546 \AA$ & F0 V-F9 V \\
N I $6596 \AA$ & \\
\hline Ca I $6162 \AA$ & F0 V-M0 V \\
Fe I $6136 \AA$ & \\
Fe I $6677 \AA$ & \\
Ca I/Fe I $6494 \AA$ & \\
\hline
\end{tabular}

parameter can also be obtained by estimating, if possible, the absolute magnitude $M_{V}$ of each star, and then comparing it with standard values of that magnitude for standard stars of different luminosity classes. One must be cautious with the results obtained following this method since we are comparing magnitudes of stars with peculiar features with magnitudes of standard, normal stars. $M_{V}$ can be easily found from $V$ and the distance (parallax), provided the colour excess $E(B-V)$ is known. A total of 38 stars have parallaxes available from Hipparcos, these have been retrieved from $\mathrm{SIMBAD}^{2}$.

\subsection{Spectral ranges and lines used in the classification}

In general, the comparison between the spectra of the target stars and the standards has made use of all the lines present, excluding only those whose origin was clearly circumstellar or interstellar. Particular care was taken in excluding the so-called DIB's (diffuse interstellar bands) at the following wavelengths: 5780, 5797, 5850, 6196, 6234, 6270, 6284, 6369, 6376 and $6614 \AA$ (Schmidt-Kaler 1982; Moutou et al. 1999).

In Table 4 we list some specific lines especially useful for classifying late-type stars ( $F$ to $M$ ) due to their gradual variation in intensity as one moves from earlier to later spectral types. We show in the table the interval of spectral types where the changes in intensity are significant for discriminating between similar types. In addition to these lines, the molecular bands $\mathrm{TiO}$ and $\mathrm{VO}$ appear in the spectra of late-type stars cooler than K7 V and their depth increases for later spectral types. Further information on lines useful for classifying this kind of object in other spectral intervals can be found in Cohen \& Kuhi (1979), Schmidt-Kaler (1982) and Jaschek \& Jaschek (1990).

For hot stars (B and A) the He I lines at 5875 and $6678 \AA$ have been used to confirm the $\mathrm{B}$ types when these lines are clearly photospheric, i.e., when no significant changes were observed when comparing spectra of the same star obtained on different dates. The presence of variability in these lines probably indicates that they

\footnotetext{
${ }^{2}$ http://simbad.u-strasbg.fr
}

are formed in the circumstellar environment and therefore they are not appropriate for classification.

\subsection{Sources of errors in the spectral classification}

The classification is based on comparisons of the target spectra with those of standards broadened with the rotational profile corresponding to the target star. Therefore, when the projected rotational velocity is of the order of $200 \mathrm{~km} \mathrm{~s}^{-1}$ or larger, the lines in the spectra are blended and it is not possible to carry out a comparison line by line of both sets of spectra, although a comparison of the broad features is still feasible. In that case, it is possible to give an estimate of the spectral type but with around five subtypes of error; the use of IUE spectra could help to classify these stars, since the shape of the continuum is not very much affected by the high rotational velocity, although, as we mention in Sect. 4.1, the lack of simultaneity between the ultraviolet and optical observations could lead to the assignment of different spectral types if some kind of variability is present. The uncertainties in the fitting of Kurucz's synthetic models to the ultraviolet spectra arise mainly from the fact that some ultraviolet excess may be present, as it is observed in some $\mathrm{T}$ Tauri and Herbig Ae/Be stars. In these cases we have not attempted any fit, thus avoiding a wrong classification of the star.

Another source of error in the determination of the spectral type of a young star is the potential variability in the equivalent width and shape of the lines and even the appearance and disappearance of some lines in intervals of a few days due to intense circumstellar activity. Therefore, it is very important to have several spectra of the same star, obtained with enough separation in time to distinguish which lines are photospheric and which are formed in the circumstellar environment.

\subsection{The problem of veiling}

The phenomenon of veiling can be defined as the superposition of a continuum on the stellar spectrum proper. The interpretation of this phenomenon is a matter of debate and different scenarios have been proposed in the last few years. It is not whithin the scope of this paper to discuss which one is the most physically sound.

It is clear that this effect must be taken into account when attempting to classify a PMS star. A star with strong veiling would have spectral lines attenuated with respect to the global continuum, therefore one would tend to use standard stars corresponding to an earlier spectral type - compared with the real one - since these show fewer and less intense lines than the spectra of late-type templates.

In a first step of our work, five stars in the sample, namely HR 26 B, HR 5422 B, BO Cep, CW Tau and DR Tau, were classified with spectral types much earlier than those reported in previous studies. This led us to consider the effect of veiling in their spectra. DR Tau has 
Table 5. Veiling values from INT spectra.

\begin{tabular}{ll}
\hline Star & Veiling \\
\hline HR 26 B & $0.1 \pm 0.1$ \\
HR 5422 B & $0.3 \pm 0.1$ \\
BO Cep & $1.0 \pm 0.4$ \\
CW Tau & $0.7 \pm 0.4$ \\
DR Tau (Oct) & $1.0 \pm 0.5$ \\
DR Tau (Jan) & $3.0 \pm 1.0$ \\
\hline
\end{tabular}

been studied extensively and a strong veiling has been reported, as we explain in detail below; CW Tau shows veiling in the near infrared range (Folha \& Emerson 1999). For HR 26 B, HR 5422 B and BO Cep, it turned out that a classification taking into account veiling effects was much more accurate. In Table 5 we give the values for the veiling we have found for these five stars with their correspondent uncertainties. Note the variability in DR Tau from October 1998 to January 1999.

Hessman \& Guenther (1997) give a detailed description of how the veiling can be parametrized (see Sect. 4 of their paper). We have used as a working definition of veiling the ratio between the non-stellar to the stellar continuum. In order to classify the stars with veiling we have added a flat continuum to the spectra of the grid of standards with several values of the veiling, in the sense described above, and normalizing afterwards to unity again. Given the subtle difference among these spectra, it is impossible to decide only by visual inspection which one is the closest to the target spectrum. To overcome this difficulty we have carried out cross correlations between the target spectrum and the grid of standard stars, both unaffected and affected by veiling, in order to determine the correct spectral type.

We show in Fig. 5 two examples of this process. In the upper panel the average INT spectrum of DR Tau obtained in October 1998 (thick line) is compared with both the spectrum of a $\mathrm{K} 5 \mathrm{~V}$ standard affected by a veiling of 1.0 (i.e. a flat continuum has been added to the spectrum with the same level as the stellar continuum), and the spectrum of a F6 V standard which could lead to erroneous classification if veiling is not considered. The three spectra are normalized and the standards have been shifted downwards for clarity. At the very top of the graph the spectrum of DR Tau obtained in January 1999 is shown, shifted upwards. A similar comparison is done for the star HR $26 \mathrm{~B}$ in the lower panel. It can be seen how subtle the differences are, especially in the case of $\mathrm{HR} 26 \mathrm{~B}$, which can easily lead, if one is not careful enough, to a wrong determination of the spectral type.

DR Tau is one of the classic cases of a star with veiling. Basri \& Batalha (1990), Guenther \& Hessman (1993) and Hessman \& Guenther (1997) studied this object and reported a large veiling effect in the spectrum. The object is highly variable; Hessman \& Guenther reported a veiling of 5.58 from observations taken in 1991, whereas in this work a veiling of 1.0 has been determined for the October 1998 observation and a higher value, around 3.0, is estimated for the January 1999 spectrum. The weakening of the absorption lines between the two epochs is apparent in the spectra of DR Tau shown in Fig. 5, accompanied by an enhancement in some of the emission lines, and even the appearance in emission of the $\mathrm{Na}$ I doublet.

\subsection{Results of the spectral classification}

We have very accurately determined the spectral type of the 70 stars observed with the INT. Out of them, 58 stars (83\%) have been classified with an error less than two spectral subtypes and $12(17 \%)$ with an error of up to five spectral subtypes. 31 stars $(43 \%)$ now have a new spectral type from our work, this means that the former spectral types are outside the error bars of our new determination or that we have different or new information about the luminosity class.

In Fig. 6 we show some representative examples of the spectral classification of several types of stars in the sample. From top to bottom we can see the spectra of BD+31 643 (Vega type), HR 10 (A-shell star), HD 190073 (Herbig Ae/Be), CO Ori (early T Tauri) and LkH $\alpha 200$ (classical T Tauri) (thick lines) and the spectra of the standards, broadened with the corresponding rotation profiles, (thin lines, shifted 0.35 units down in the intensity scale), that give the best fits to the target spectra.

In Table 6 we give the result of the spectral classification for the 70 target stars. Column 1 is the star identification, Col. 2 gives the class of star, in Cols. 3 and 4 we give the result of our classification and those from previous work, respectively. The class of star (Col. 2) for each object has been assigned according to the spectral types found in this work. We have adopted the criterion of assigning a main-sequence type to the primary stars of the Lindroos' binaries and a PTT type to the secondary stars.

Almost all the PMS in the sample present spectral variability. Some non-photospheric features appearing in the spectra are: $\mathrm{H} \alpha$ in emission, forbidden emission lines ([O I] $6300 \AA$, [O I] $6363 \AA$, [S II] $6716 \AA$, [S II] $6731 \AA$, [N II] $6583 \AA,[\mathrm{N} \mathrm{II}] 6548 \AA$ and [N II] $6527 \AA$ ) which may be related to winds; and variable lines of He I $5875 \AA, 6678 \AA$ and $\mathrm{Na} \mathrm{I}_{1} \mathrm{D}_{2} 5890,5896 \AA$ that may be produced in the circumstellar environment.

\subsection{Comments on particular stars}

In this subsection we comment on the stars marked with an asterisk in Table 6 and also on those showing some peculiarity.

HD 58647, HD 141569, HD 163296, HR 2174 and VX Cas have very high projected rotational velocities and show many non-photospheric features in their spectra. VY Mon also shows a lot of circumstellar or interstellar lines contaminating its spectrum; V1686 Cyg and VV Ser show very variable emission lines; MWC 297 has very few useful lines in absorption for classification due 


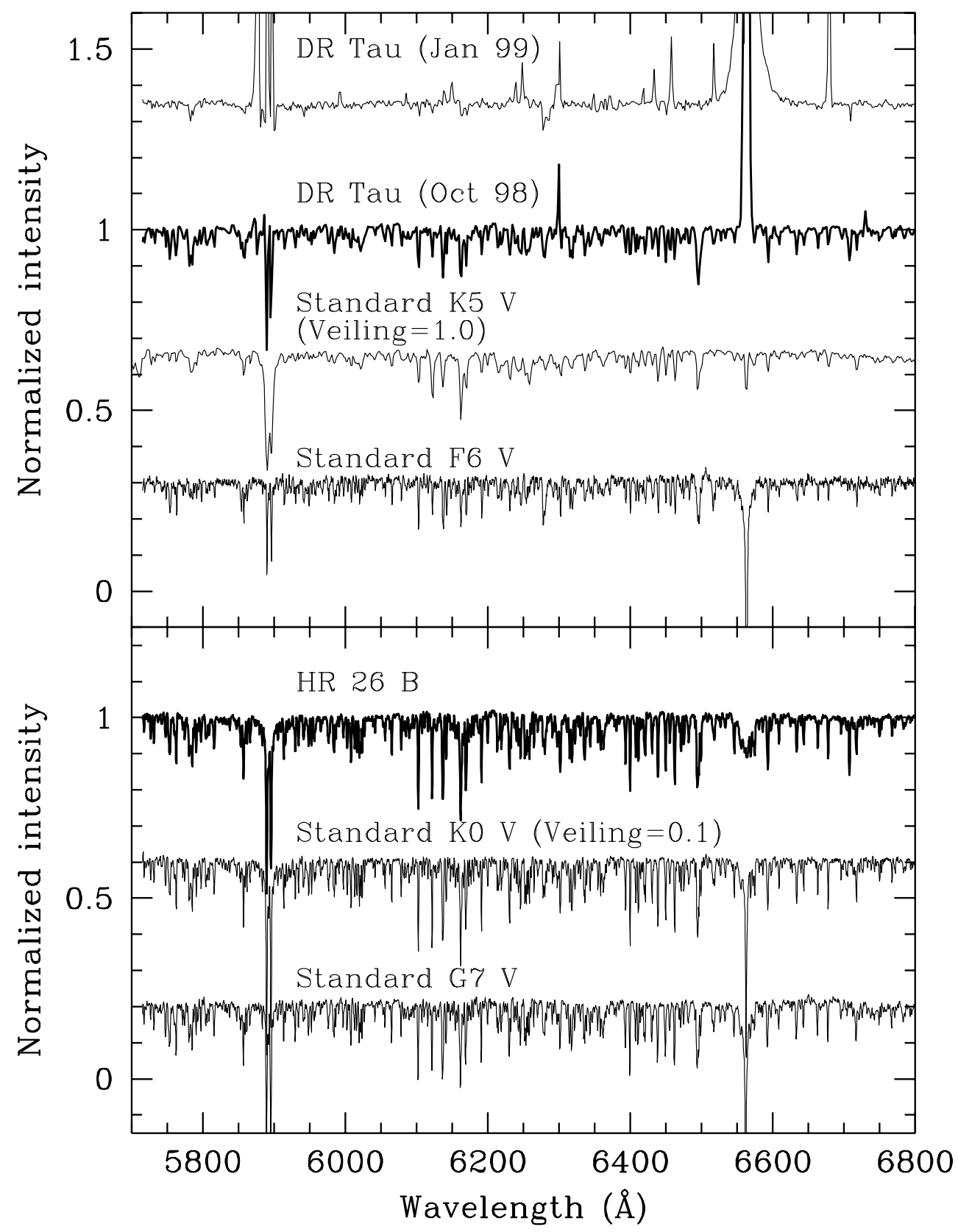

Fig. 5. Two examples of classification of stars with veiling. Upper panel: from top to bottom, the spectra of DR Tau obtained in January 1999 and October 1998, the spectrum of a standard K5 V with a veiling of 1.0 (see text for details) and a standard F6 V, which was the first choice for the spectral type before veiling was considered. Lower panel: the spectra of HR $26 \mathrm{~B}$, a standard $\mathrm{K} 0 \mathrm{~V}$ with a veiling of 0.1 and the spectrum of a $\mathrm{G} 7 \mathrm{~V}$ standard.

to the blends caused by its high $v \sin i\left(350 \pm 50 \mathrm{~km} \mathrm{~s}^{-1}\right.$, Drew et al. 1997); HD 123160, RR Tau and $\mathrm{LkH} \alpha 262$ show changes in the continuum in time spans of days to months; HR 26 B, HR 5422 B, BO Cep, CW Tau and DR Tau show veiling that attenuate the absorption lines.

In addition, HK Ori shows a composite spectrum from a non-resolved binary system; HR 10 has an INT spectrum classified as a A0 Vn but shows an IUE spectrum only compatible with a G1 V Kurucz model.

HD 23362 and HD 233517, thought to be Vega-type stars, are now classified according to our analysis as K-type giant stars and under the hypothesis that they are
PMS stars, they should be considered as CTT objects. However, this classification must be taken with caution; Castilho et al. (1998) and Balachandran et al. (2000) classify these stars as post-main sequence giants, with the peculiarity of both being Lithium rich.

\section{The projected rotational velocities}

One of the major gaps in our understanding of stellar physics is the role of angular momentum in the formation and early life of a star. Two problems of great interest are the transport of angular momentum in collapsing 


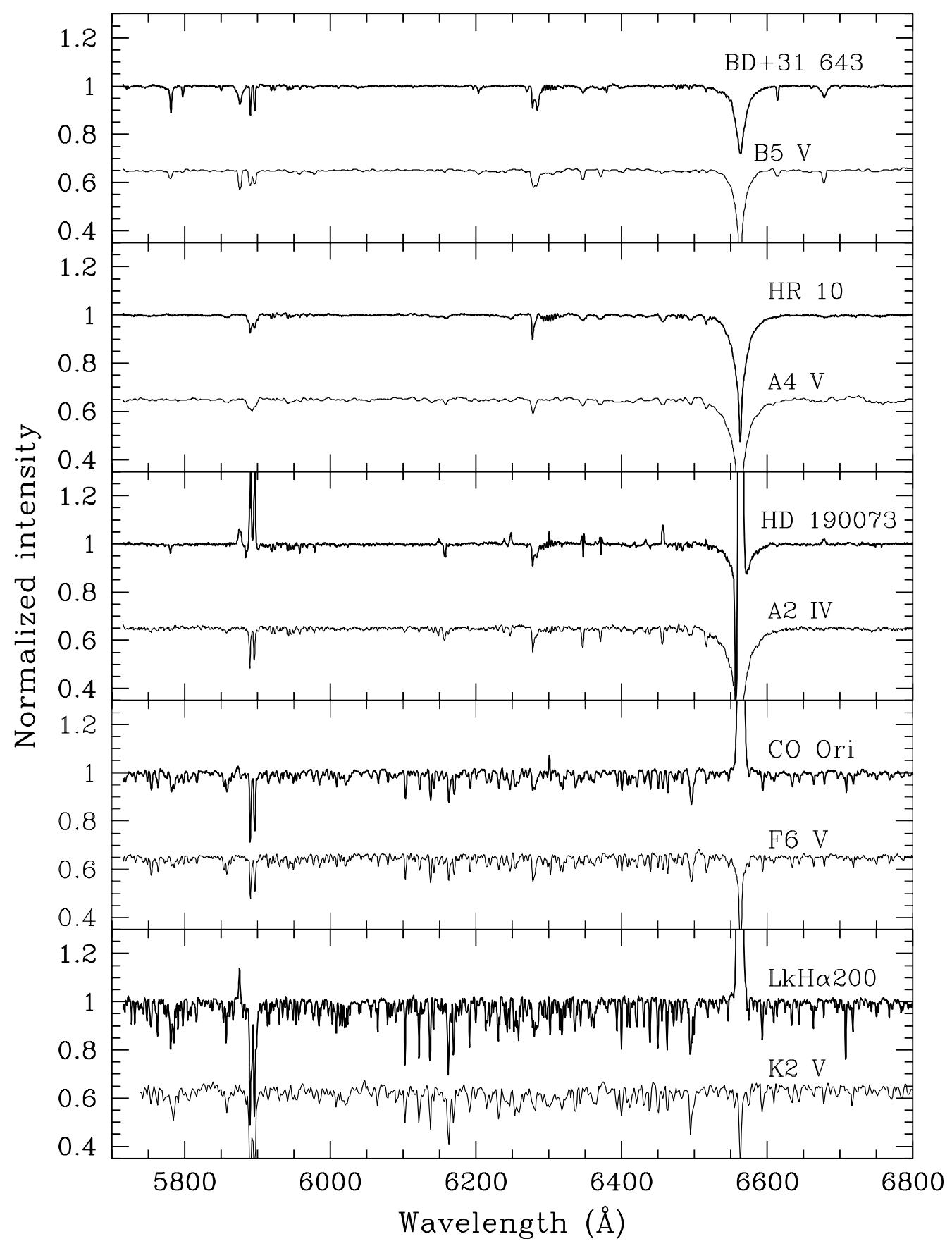

Fig. 6. Five examples of the final results of the spectral classification. The spectra of the target stars are drawn with thicker lines, the spectra of the standards have been shifted down 0.35 units for clarity. From top to bottom, BD+31 643 (Vega type) and the standard HR 930 (B5 V) broadened with $v \sin i=162 \mathrm{~km} \mathrm{~s}^{-1}$, HR 10 (A-shell) and the standard HR 4633 (A4 V) broadened with $v \sin i=110 \mathrm{~km} \mathrm{~s}^{-1}$, HD 190073 (Herbig Ae/Be) and the standard HR 4670 (A2 IV), CO Ori (ETT) and the standard HR 4606 (F6 V), and LkH $\alpha 200$ (CTT) and the standard HD 166620 (K2 V).

interstellar clouds and the subsequent braking of young stars during the PMS contraction. Accurate rotational velocities of young stars are essential for further progress in these areas. Also, the knowledge of the behaviour of the distribution of rotational velocities as a function of the spectral type puts important constraints on models of the stellar angular momentum evolution and provides information on what physical process controls the rotational velocity across the HR diagram.
Different methods have been developed to calculate rotational velocities, all of them relying, to some extent, on the geometrical technique, suggested originally by Shajn \& Struve (1929), that relates line profiles and line widths to the apparent rotational velocity, $v \sin i$. Until the advent of solid-state detectors about two decades ago, most of the rotational velocities were determined by measurements of one or two relatively strong lines. The method consisted in identifying a particular point on the line profile (usually 
Table 6. Results of the spectral classification and projected rotational velocities. The values of $v \sin i$ are given in $\mathrm{km} \mathrm{s}^{-1}$.

\begin{tabular}{|c|c|c|c|c|c|}
\hline Star & Type of star & Spectral type & Previous classifications & $v \sin i$ & $\begin{array}{l}v \sin i \\
\text { Previous work }\end{array}$ \\
\hline HD 23362 & CTT & K5 IIIm & $\mathrm{K} 2 \mathrm{~V}, \mathrm{~K} 2$ & $6 \pm 1$ & \\
\hline HD 23680 & CTT & G5 IV & G5 & & \\
\hline HD 31293 & & & & $97 \pm 20$ & $80 \pm 5^{(15)}$ \\
\hline HD 31648 & $\mathrm{HAeBe}$ & A5 Vep & A3ep+sh, A3ep+sh, A2 & $102 \pm 5$ & $80^{(1)}$ \\
\hline HD 34282 & $\mathrm{HAeBe}$ & A3 Vne & $\mathrm{A} 2 \mathrm{Ve}+\mathrm{sh}, \mathrm{A} 0 \mathrm{e}, \mathrm{A} 0$ & $129 \pm 11$ & \\
\hline HD 34700 & ETT & G0 IVe & G0 V & $46 \pm 3$ & \\
\hline HD $58647^{*}$ & $\mathrm{HAeBe}$ & B9 IVep & A0 IVe, B9 II-IIIe, B9e & $118 \pm 4$ & \\
\hline HD 109085 & Vega & $\mathrm{F} 2 \mathrm{~V}$ & F2 V, F3 V, F2 III-IV & $68 \pm 2$ & $51^{(2)}, 81^{(3)}$ \\
\hline HD $123160^{*}$ & CTT & K5 III & G5 V, K5 & $7.8 \pm 0.5$ & $9 \pm 1^{(4)}$ \\
\hline HD 141569 & $\mathrm{HAeBe}$ & A0 Vev & A0 Ve, B9.5 Ve, B9e & $258 \pm 17$ & $236 \pm 9^{(4)}$ \\
\hline HD 142666 & HAeBe & $\mathrm{A} 8 \mathrm{Ve}$ & $\mathrm{A} 7 \mathrm{~V}, \mathrm{~A} 8 \mathrm{~V}, \mathrm{~A} 8 \mathrm{Ve}$ & $72 \pm 2$ & $70 \pm 2^{(4)}$ \\
\hline HD 142764 & Vega & $\mathrm{K} 7 \mathrm{~V}$ & K5 & $7.8 \pm 1.5$ & \\
\hline HD 144432 & $\mathrm{HAeBe}$ & A9 IVev & $\mathrm{A} 7 \mathrm{Ve}, \mathrm{A} 9 / \mathrm{F} 0 \mathrm{~V}, \mathrm{~A} 7 \mathrm{Ve}$ & $85 \pm 4$ & $74 \pm 2^{(4)}, 73^{(5)}$ \\
\hline HD 150193 & $\mathrm{HAeBe}$ & A2 IVe & $\mathrm{A} 2 \mathrm{Ve}, \mathrm{A} 1 \mathrm{~V}, \mathrm{~A} 1 \mathrm{Ve}$ & & \\
\hline HD 158352 & MS & $\mathrm{A} 8 \mathrm{Vp}$ & $\mathrm{A} 7 / 8 \mathrm{~V}+\mathrm{sh}, \mathrm{A} 8 \mathrm{Vsh}$ & & \\
\hline HD 163296 & $\mathrm{HAeBe}$ & A1 Vepv & A3 Vep+sh, A1 V, A0-A2 & $133 \pm 6$ & $120^{(6)}$ \\
\hline HD 179218 & $\mathrm{HAeBe}$ & A0 IVe & $\mathrm{B} 9 / \mathrm{A} 0 \mathrm{IV} / \mathrm{Ve}, \mathrm{B} 0 \mathrm{e}$ & & \\
\hline HD 190073 & $\mathrm{HAeBe}$ & A2 IVev & B9/A0 Vp+sh, A0 IV esh & & \\
\hline HD 199143 & $\mathrm{MS}$ & $\mathrm{F} 6 \mathrm{~V}$ & F6 V & $155 \pm 8$ & \\
\hline HD 203024 & MS & A $5 \mathrm{~V}$ & $\mathrm{Ae}$ & & \\
\hline HD 233517 & $\mathrm{CTT}$ & K5 III & $\mathrm{K} 2, \mathrm{~A} 2$ & $16 \pm 1$ & $15^{(7)}, 17.6^{(19)}$ \\
\hline HR 10 & Ash & $\mathrm{A} 0 \mathrm{Vn}$ & $\mathrm{A} 2 \mathrm{~V}-\mathrm{A} 5 \mathrm{~V}, \mathrm{~A} 6 \mathrm{Vn}$ & $294 \pm 9^{\dagger}$ & $220^{(8)}, 195^{(9)}$ \\
\hline $\mathrm{HR} 26 \mathrm{~A}$ & MS & B9 Vn & B9 Vn, B8.5 Vnn & $266 \pm 5$ & $275^{(10)}$ \\
\hline HR $26 \mathrm{~B}$ & PTT & $\mathrm{K} 0 \mathrm{~V}$ & G5 Ve & & \\
\hline HR 419 & & & & $162 \pm 14$ & \\
\hline HR 1847 & Vega & B5 V & B7 IIIe & & \\
\hline HR $2174^{*}$ & Vega & $\mathrm{A} 2 \mathrm{Vnv}$ & A3 Vn, A1 IV-sh, A3 V & $252 \pm 7$ & \\
\hline HR $4757 \mathrm{~A}$ & MS & B9.5 V & $\mathrm{B} 9.5 \mathrm{~V}$ & $239 \pm 7$ & $205^{(11)}$ \\
\hline HR $4757 \mathrm{~B}$ & PTT & $\mathrm{K} 2 \mathrm{~V}$ & $\mathrm{~K} 0 \mathrm{~V}, \mathrm{~K} 2 \mathrm{Ve}, \mathrm{K} 1 \mathrm{~V}$ & $5.7 \pm 0.7$ & \\
\hline HR 5422 A & MS & $\mathrm{A} 0 \mathrm{~V}$ & A0 V, B9.2p, B9 Vp & $7.4 \pm 0.3$ & $14^{(12)}, 20^{(13)}$ \\
\hline HR $5422 \mathrm{~B}$ & PTT & $\mathrm{K} 0 \mathrm{~V}$ & $\mathrm{~K} 1 \mathrm{~V}$ & & \\
\hline HR 9043 & Vega & A5 Vn & A5 V, A3 Vn & $205 \pm 18$ & $210^{(8)}$ \\
\hline AS 442 & HAeBe & $\mathrm{B} 8 \mathrm{Ve}$ & $\mathrm{B} 8 \mathrm{e}$ & & \\
\hline $\mathrm{BD}+31643$ & Vega & B5 V & $\mathrm{B} 5, \mathrm{~B} 5 \mathrm{~V}$ & $162 \pm 13^{\dagger}$ & \\
\hline MWC $297^{*}$ & $\mathrm{HAeBe}$ & $\mathrm{B} 1 \mathrm{Ve}$ & O9e, Be, B1.5, 09 & & \\
\hline BM And & CTT & $\mathrm{K} 5 \mathrm{Ve}$ & $\mathrm{K} 5 \mathrm{~V}$ & & \\
\hline$\lambda \mathrm{Boo}$ & Vega & $\mathrm{A} 1 \mathrm{~V}$ & A0 Vpsh, A0p & $129 \pm 7$ & $100^{(14)}$ \\
\hline VX Cas & $\mathrm{HAeBe}$ & A0 Vep & $\mathrm{A} 0 / 3 \mathrm{e}, \mathrm{A} 0, \mathrm{~A} 3, \mathrm{~A} 0 \mathrm{e}$ & $179 \pm 18$ & \\
\hline BH Cep & ETT & F5 IIIev & A/F5 Ve, F5 IVvar & $98 \pm 3$ & \\
\hline BO Cep & CTT & F5 Ve & F2e, F2e & & \\
\hline SV Cep & $\mathrm{HAeBe}$ & A2 IVe & $\mathrm{A} 0 \mathrm{e}, \mathrm{A}$ & $206 \pm 13$ & \\
\hline 49 Cet & Vega & $\mathrm{A} 4 \mathrm{~V}$ & $\mathrm{~A} 3 \mathrm{~V}, \mathrm{~A} 1 \mathrm{~V}$ & $186 \pm 4$ & \\
\hline $24 \mathrm{CVn}$ & Ash & $\mathrm{A} 4 \mathrm{~V}$ & $\mathrm{~A} 5 \mathrm{~V}, \mathrm{~A} 4 \mathrm{~V}, \mathrm{~A} 5.5 \mathrm{~A}$ & $173 \pm 4$ & $145^{(3)}, 160^{(1)}$ \\
\hline V1685 Cyg & $\mathrm{HAeBe}$ & $\mathrm{B} 2 \mathrm{Ve}$ & $\mathrm{B} 2 \mathrm{Ve}+\mathrm{sh}, \mathrm{B} 2, \mathrm{~B} 2 \mathrm{Ve}$ & & \\
\hline V1686 Cyg* & $\mathrm{HAeBe}$ & $\mathrm{A} 4 \mathrm{Ve}$ & $\mathrm{G} 2 \mathrm{~V}, \mathrm{~A} 0 \mathrm{~V}, \mathrm{~A} 7 \mathrm{~V}$ & & \\
\hline R Mon & $\mathrm{HAeBe}$ & B8 IIIev & B0e, B.., B2 & & \\
\hline VY Mon* & $\mathrm{HAeBe}$ & A5 Vep & $\mathrm{B} 9 / \mathrm{A} 0 \mathrm{e}, \mathrm{B} 8, \mathrm{~B}-\mathrm{A}$ & & \\
\hline $51 \mathrm{Oph}$ & $\mathrm{HAeBe}$ & B9.5 IIIe & A0 V, B9.5 Ve,A0 II-III(e) & $256 \pm 11^{\dagger}$ & $267^{(4)}$ \\
\hline KK Oph & $\mathrm{HAeBe}$ & A8 Vev & $\mathrm{B} 3, \mathrm{~B}-\mathrm{Ae}, \mathrm{A} 5 \mathrm{Ve}, \mathrm{A} 6$ & $177 \pm 13^{\dagger}$ & \\
\hline T Ori & $\mathrm{HAeBe}$ & A3 IVev & $\mathrm{A} 3 / 4 \mathrm{e}, \mathrm{A} 3, \mathrm{~B} 9, \mathrm{~A} 5 \mathrm{e}$ & $175 \pm 14$ & $130 \pm 20^{(15)}$ \\
\hline BF Ori & $\mathrm{HAeBe}$ & A2 IVev & A5/6 IIIe+sh, A5e, A6e & $37 \pm 2$ & $100^{(15)}$ \\
\hline CO Ori & $\mathrm{ETT}$ & F7 Vev & F9/G Ve, F9: e, F8 & $65 \pm 4$ & $48 \pm 15^{(16)}$ \\
\hline HK Ori* & ETT & G1 Ve & $\mathrm{A} 5, \mathrm{~A} 4, \mathrm{G}: \mathrm{ep}, \mathrm{B} 8 / \mathrm{A} 4 \mathrm{ep}$ & & \\
\hline NV Ori & ETT & F6 IIIev & F0/8 IVe, F 4/0 III,V & $81 \pm 8$ & $80 \pm 7^{(15)}$ \\
\hline RY Ori & ETT & F6 Vev & F6/Gep, F8:pe & $66 \pm 6$ & \\
\hline
\end{tabular}


Table 6. continued.

\begin{tabular}{llllll}
\hline Star & Type of star & Spectral type & Previous classifications & $v \sin i$ & $\begin{array}{l}v \sin i \\
\text { Previous work }\end{array}$ \\
\hline UX Ori & HAeBe & A4 IVe & A3e, A2/3 IIIe, A1-3IIIe & $215 \pm 15$ & $175^{(17)}, 70 \pm 6^{(15)}$ \\
V346 Ori & HAeBe & A2 IV & F1:III:e, A5 III:e & & \\
V350 Ori & HAeBe & A2 IVe & A5e, A0 & \\
XY Per & HAeBe & A2 IV & B6/A5e, B6, A2 II+B6e & $217 \pm 13$ & \\
VV Ser* & HAeBe & A0 Vevp & A2e, B1-3e:, B1/3e/A2: & $229 \pm 9^{\dagger}$ & \\
17 Sex & Ash & A0 V & A1 V, A1 Vsh, A5 & $259 \pm 13^{\dagger}$ & $180^{(3)}$ \\
CQ Tau & ETT & F5 IVe & F2:e, A8 IV /F2 IVe & $105 \pm 5$ & $110 \pm 20^{(15)}$ \\
CW Tau* & CTT & K3 Ve & K3, K5 V:e & & \\
DK Tau & CTT & K5 Ve & M0 V:e, K7 V, K7 & & \\
DR Tau* & CTT & K5 Vev & K4 V:e & & \\
RR Tau* & HAeBe & A0 IVev & F:e, A2 II-IIIe, B8ea & $225 \pm 35^{\dagger}$ & \\
RY Tau & ETT & F8 IIIev & F8 V:e, K1 IV, G5e, K7 & $55 \pm 3$ & $52^{(18)}$ \\
PX Vul & ETT & F3 Ve & F0 V:e, F0, F5 & & \\
WW Vul & HAeBe & A2 IVe & A3e, A0/3 Ve, A0, A1e & $220 \pm 22$ & \\
LkH $\alpha 200$ & CTT & K3 Ve & K1 V, Ke, dK0 & & \\
LkH $\alpha 234$ & HAeBe & B5 Vev & B5.7e, B5/7, B9/A0e, A7 & & \\
LkH $\alpha 262 *$ & CTT & M1 IIIe & K?, M0 & & \\
\hline
\end{tabular}

Notes to Table 6: The meanings of the lower case suffixes are: "e" emission lines, "v" variable spectrum, "p" peculiar spectrum (presence of non-standard components), " $\mathrm{n}$ " broad or weak lines in the spectrum, "m" unexpected lines from metals or metal lines with unusually large strengths.

The abbreviations in Col. 2 mean: CTT (classical T Tauri), ETT (early T Tauri), HAeBe (Herbig Ae/Be), MS (main sequence), PTT (Post-T Tauri) and Ash (A-shell star). The stars marked with an asterisk $\left(^{*}\right)$ have been classified with an error of about five spectral subtypes because they present peculiar spectra, too many diffuse interstellar bands or veiling. In Sect. 4.6 we give particular details on these stars.

A blank in Col. 5 means that the star was not observed with the WHT, furthermore no determination of $v \sin i$ was feasible. The symbol $\dagger$ indicates that $v \sin i$ has been obtained using only the Mg II $4481 \AA$ line.

References to $v \sin i$ in Col. 6: (1) Jaschek et al. (1988); (2) Wolff \& Simon (1997); (3) Abt \& Morrel (1995); (4) Dunkin et al. (1997); (5) Fekel (1997); (6) van den Ancker et al. (1998); (7) Jasniewicz et al. (1999); (8) Welsh et al. (1998); (9) Lecavelier des Etangs et al. (1997); (10) Ghosh et al. (1999); (11) Baade (1989); (12) Ramella et al. (1989); (13) Millward \& Walker (1985); (14) Paunzen et al. (1999); (15) Böhm \& Catala (1995); (16) Fernández \& Miranda (1998); (17) Grady et al. (1996); (18) Petrov et al. (1999); (19) Balachandran et al. (2000).

the full width at half maximum) and calibrating this parameter in terms of a rotational standard. Although helpful in identifying older sources of $v \sin i$ measurements, catalogues such as those of Bernacca \& Perinotto (1970) and Uesugi \& Fukuda (1982) pose serious problems because they attempt to combine observations that have different resolutions obtained with a variety of techniques.

The use of modern detectors and the associated improvement of both spectral resolution and signal-to-noise ratio has permitted the use of more accurate techniques: methods based on comparisons between the observed spectrum and synthetic spectra convolved by instrumental and rotational broadening functions (e.g. Magee et al. 1998) or cross-correlation analysis (e.g. Tonry \& Davis 1979) have become widespread.

In this paper, we make use of the technique proposed by Gray (1992). In short, the method is based on the relation between $v \sin i$ and the frequencies where the Fourier transform of the rotational profile reaches a relative minimum: the dominant term in the Fourier transform of the rotational profile is a first-order Bessel function that produces a series of relative minima at regularly spaced frequencies (Fig. 7). Unlike the above-mentioned methods which require the building up of a calibration library of rotational velocities, Gray's method provides a direct measurement of $v \sin i$. Moreover, it also allows the differentiation of rotation from other potential competing broadening sources such us, for instance, macroturbulence in late-type stars. Projected rotational velocities, in $\mathrm{km} \mathrm{s}^{-1}$, calculated using the high resolution spectra taken with the WHT are given in Col. 5 of Table 6 ; results from previous work are shown in Col. 6. Metallic lines of photospheric origin covering the full wavelength range and whose main source of broadening is rotation were used in this analysis.

Projected rotational velocities were not calculated for two stars of our sample: DR Tau and HK Ori (Fig. 8). DR Tau shows, in the range covered by the WHT observations, a spectrum of complex nature characterized by the absence of absorption photospheric features caused by a high degree of veiling, whereas HK Ori is a visual and spectroscopic binary showing a composite spectrum where the contribution of a companion classified as a $\mathrm{T}$ Tauri star is superimposed (Corporon \& Lagrange 1999) (see Sect. 4.6). 

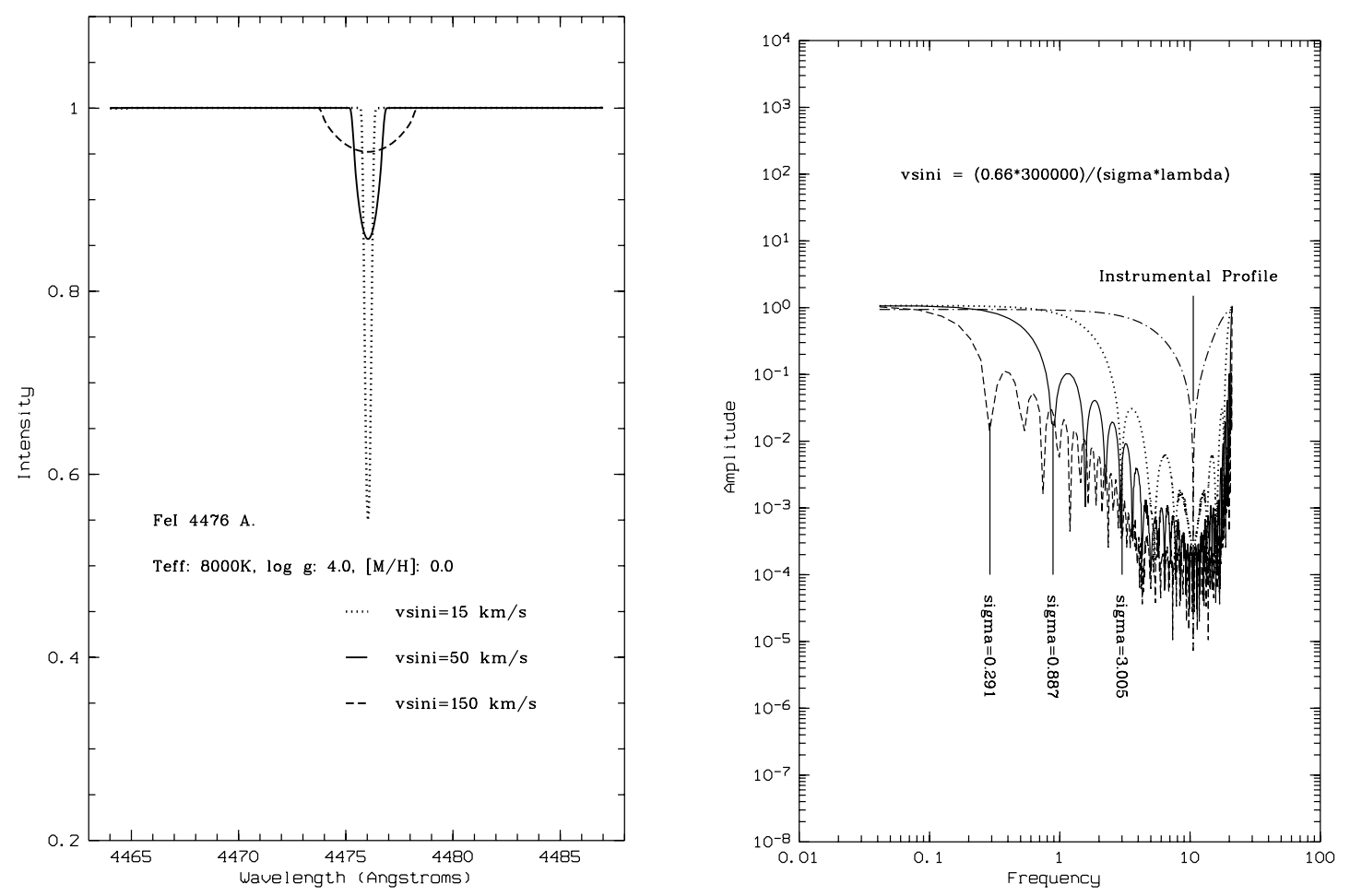

Fig. 7. Determination of projected rotational velocities via Fourier transform. Left panel: influence of rotational broadening on spectral lines. The synthetic line Fe I $4476 \AA$ is shown for different broadenings. Right panel: Fourier transforms and their relation with rotational velocities. The frequency used to calculate $v \sin i$ is indicated. The trasform of the average instrumental profile of the WHT spectra is also plotted for comparison.
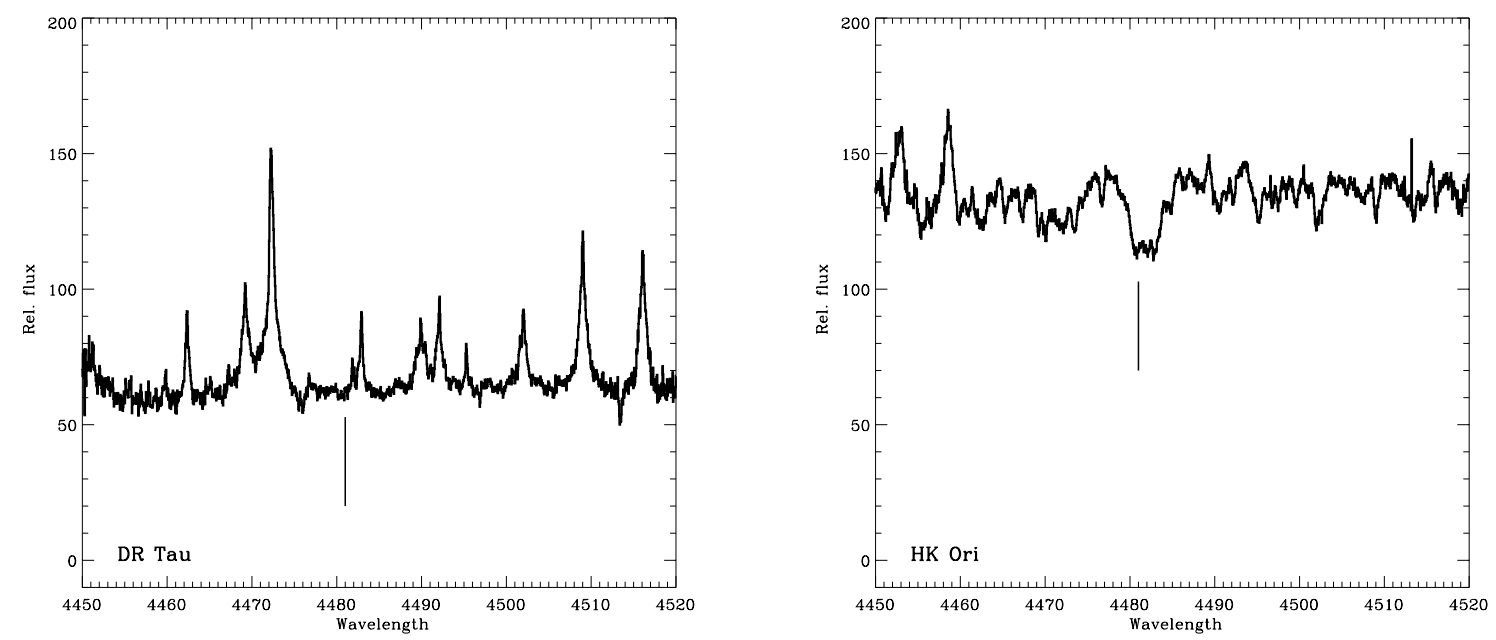

Fig. 8. The spectra of DR Tau and HK Ori. The position of the Mg II $4481 \AA$ line is shown for clarity.

In general, our results agree with those found in previous work. There are, however, some cases where clear discrepancies are apparent. Figure 9 shows those stars for which our $v \sin i$ values are clearly discrepant with previous determinations. In all cases our values produce better fits to the observations. Also, in some other cases, the blending is so severe that only one spectral line, Mg II $4481 \AA$, can be used to determine the rotational velocity. These objects are labelled with "†" in Table 6 . The Mg II $4481 \AA$ doublet is considered an ideal indicator in these cases as it is free of strong pressure broadening and yet is strong enough that the rotation-broadened profile can be easily measured. Moreover, the large rotational broadening means that the $0.20 \AA$ splitting of the doublet is not a problem.

\subsection{Estimated uncertainties}

Limb darkening: According to Eq. (17.12) of Gray (1992), one of the uncertainties in the calculation of $v \sin i$ comes from the limb-darkening law. In this work, a linear law with $\epsilon=0.6$ has been assumed. Following Díaz-Cordovés \& Giménez (1992), the difference in the total emergent flux between the linear and the quadratic 

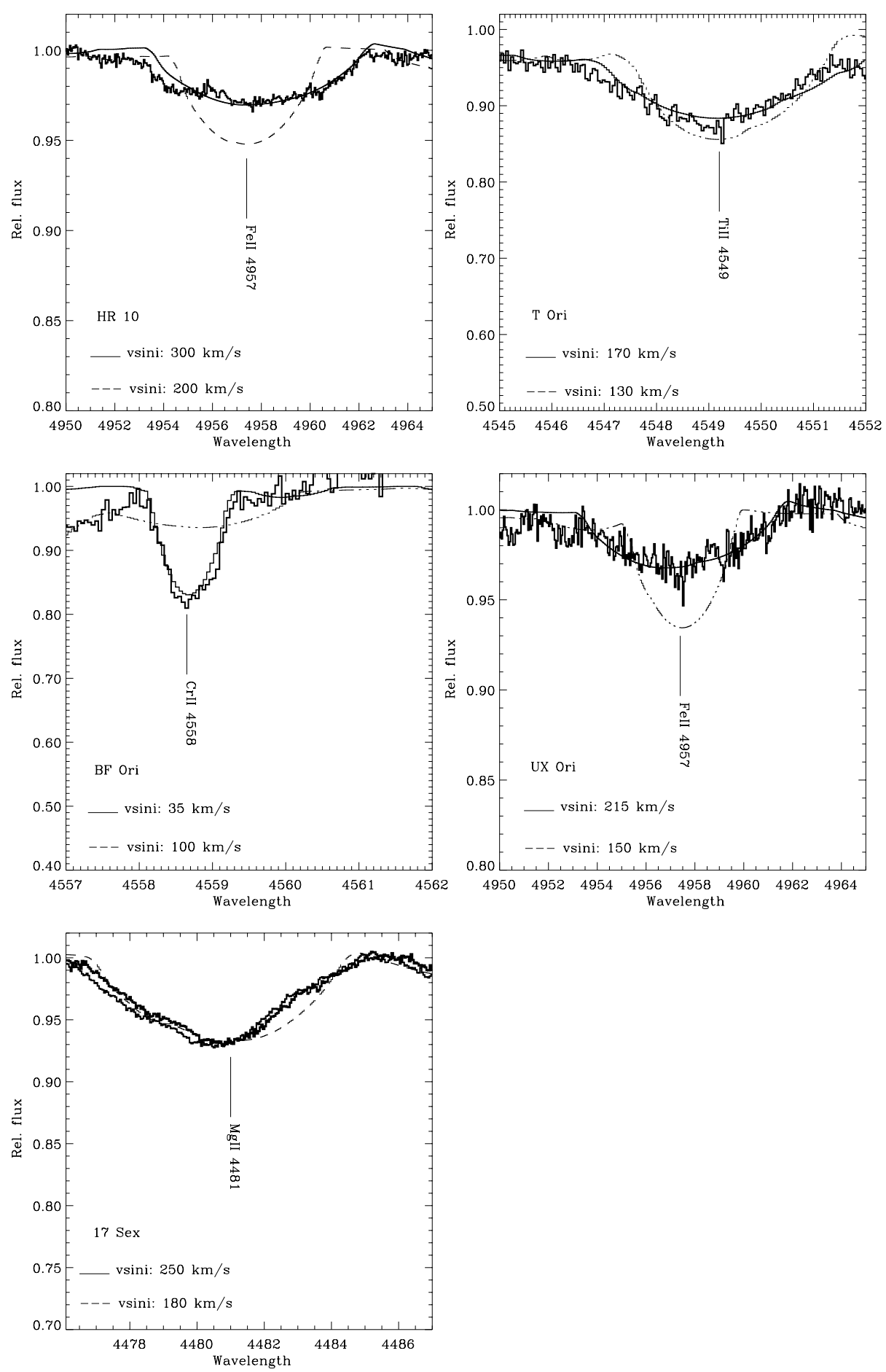

Fig. 9. Comparison between the observed spectrum and a synthetic spectrum generated with ATLAS9 (1993) convolved with the labelled rotational velocities. In all cases, our $v \sin i$ values are confirmed.

laws is less than $5 \%$ in the range of temperatures of our programme stars. Moreover, the dependence of $\epsilon$ with wavelength is also negligible: according to Díaz-Cordovés et al. (1995), we expect a variation in $\epsilon$ of $0.55 \leq \epsilon \leq 0.85$ in our range of temperatures, gravities and wavelengths. Solano \& Fernley (1997) demonstrated that such a variation implies, in the worst case, a change in $v \sin i$ of less than $5 \%$.
Continuum placement: The determination of the local continuum is another unavoidable source of error: a displacement in the continuum level can change the line profile, especially the wings, and thus distort the shape of the Fourier transform, modifying the position of its zeroes. Many objects in our sample have broad line profiles typical of high $v \sin i$ values. In this case, the continuum placement cannot be set by simply connecting the highest points in the observed spectrum since this would produce 

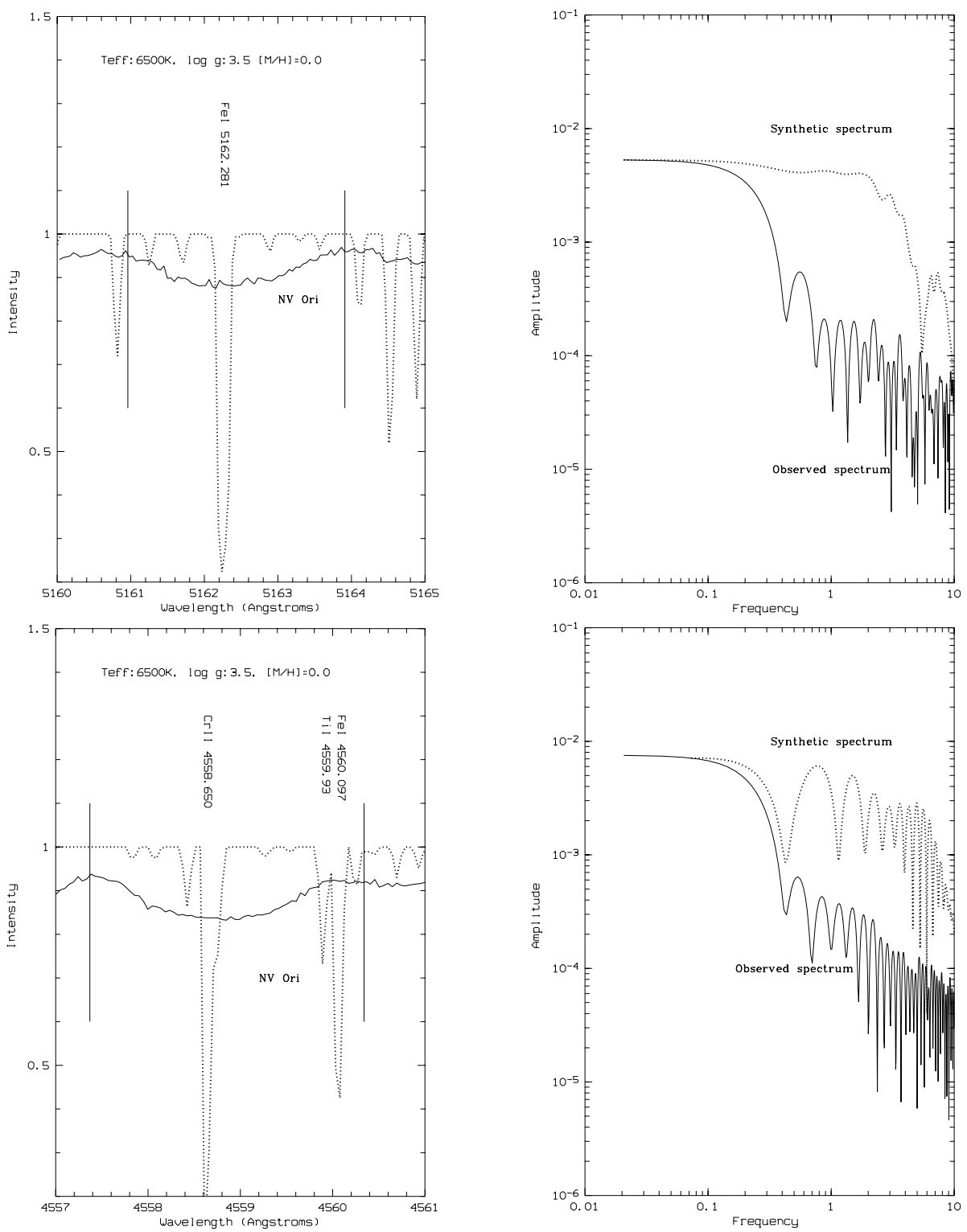

Fig. 10. Influence of the intrinsic profile of blended features on the Fourier transform. The observed spectrum (solid line) is compared to a synthetic spectrum (dotted line) with similar physical parameters $\left(T_{\text {eff }}, \log g,[\mathrm{M} / \mathrm{H}]\right)$ and $v \sin i=0$. Assumed line edges are also displayed. Top panel: the contribution of the intrinsic profile lies at high frequencies and thus does not affect the $v \sin i$ determination. Bottom panel: in this case the intrinsic profile produces a spurious minimum in the Fourier transform which may lead to a wrong $v \sin i$ determination.

an underestimation of the equivalent widths. This problem was solved by defining the "true" continuum level as that of a synthetic spectrum of physical parameters similar to those of the observed object.

Blending: The high $v \sin i$ values of many of the stars of our sample make it very difficult to apply the method to isolated lines. Blended features were used instead. A careful inspection using synthetic spectra was performed to avoid contamination in the Fourier domain due to the intrinsic profile of the blended features (Fig. 10).

Sampling frequency: Another limiting factor in the calculation of $v \sin i$ is the sampling frequency. Defining this frequency as $\sigma=0.5 / \Delta \lambda$ and considering the spectral resolution quoted in Sect. 3, a minimum value of $\approx 6 \mathrm{~km} \mathrm{~s}^{-1}$ can be achieved. Hence, for stars with $v \sin i$ lower than this value it is not possible to calculate a proper value of $v \sin i$ but only an upper limit (Table 6).

\subsection{Observed distribution of rotational velocities}

The stellar angular momentum is known to change dramatically along the evolutionary sequence. This is particularly relevant for $\mathrm{T}$ Tauri stars for which a large angular momentum loss is expected during the early stages of the star formation, where rotational velocities might decrease from values near break-up, typical of protostars, to velocities in the range from less than $10 \mathrm{~km} \mathrm{~s}^{-1}$ up to $30 \mathrm{~km} \mathrm{~s}^{-1}$ with a mean value about $15 \mathrm{~km} \mathrm{~s}^{-1}$ for a $1 M_{\odot}$ T Tauri star (Vogel \& Kuhi 1981). Within the T Tauri stars it is also possible to find statistically significant differences 


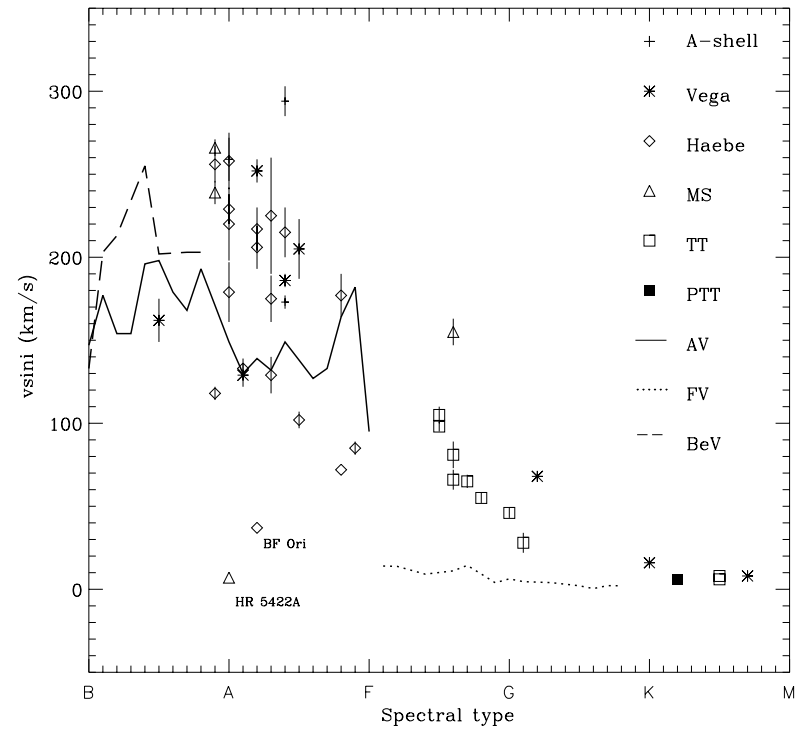

Fig. 11. Distribution of rotational velocities as a function of the spectral type.

between classical (CTTs) and weak (WTTs) T Tauri stars in the sense that the latter rotate faster suggesting a different rotational evolution (Bouvier et al. 1993). These differences can be interpreted as an evidence of the spinning-up of the WTTs as they contract while CTTs are prevented from doing so by e.g. strong winds or magnetic coupling between the star and the inner accretion disk. Solar-mass ZAMS stars in young stellar clusters represent the subsequent step in the evolutionary scenario. Contrary to TTs, these stars exhibit a much larger range of $v \sin i$, peaked at low velocities (typically $10 \mathrm{~km} \mathrm{~s}^{-1}$ ) with a wide high-velocity tail up to $200 \mathrm{~km} \mathrm{~s}^{-1}$ (Allain et al. 1996). The question of how to account for the rotational distribution of ZAMS stars starting from that of TTs is not well understood yet. Bouvier et al. (1993) proposed linking the observed rotational distribution of ZAMS stars with the two T Tauri subclasses: according to this, WTTs and CTTs would be the progenitors of the ZAMS fast and slow rotators respectively. Shortly after their arrival on the ZAMS, solar-type stars are then drastically braked and, at the age of the Hyades, (600 Myr) have rotation rates lower than $10 \mathrm{~km} \mathrm{~s}^{-1}$ irrespective of the initial angular momentum (Endal \& Sofia 1981).

The use of projected rotational velocities in the determination of the rotational properties of stars is limited by the unknown geometric effect included in $\sin i$. Moreover, the number of $v \sin i$ determinations, too low for a statistically significant analysis, is an additional limiting factor in our study. Nevertheless, it is possible to interpret the observed distribution in a qualitative way. Figure 11 displays the rotational velocities of our program stars as a function of their spectral type as given in Table 6. For comparison, we have also plotted mean rotational velocities of B, A-type stars (solid line, Fukuda 1982), F, G-type stars (dotted line, Fekel 1997) and Be stars (dashed line, Steele et al. 1999) of luminosity class V. Two stars appear to be clearly discrepant in this figure: HR 5422 A (A0 $\mathrm{V}, v \sin i=7.4 \mathrm{~km} \mathrm{~s}^{-1}$ ) and BF Ori (A2 IVev, $v \sin i=$ $37 \mathrm{~km} \mathrm{~s}^{-1}$ ). HR $5422 \mathrm{~A}$ is a Am star in a double system (Ramella et al 1989) whereas the $v \sin i$ value of BF Ori is confirmed by the large number of data used in the analysis (four spectra in two different observing runs with 8, 12, 15 and 13 lines used respectively) which suggests that the low $v \sin i$ value may be due to an inclination effect.

One of the results that can be deduced from Fig. 11 is that the PMS stars tend to rotate faster than stars in the main sequence with similar spectral types. The qualitative behaviour in the overall distribution of $v \sin i$ for pre-main sequence and main sequence stars is similar, namely, rotation is large for early-type stars dropping rapidly through the F-stars region. In the main sequence stars, this behaviour is tied in with the appearance of convective envelopes and the magnetic brake generated by its interaction with rotation in the dynamo process. Dudorov et al. (1994) proposed a similar hypothesis for PMS stars where the sudden transition in spin rates at F-spectral types would also occur as a consequence of the onset of a magnetic field in the star envelopes. Figure 11 also confirms the basic conclusions from Finkenzeller (1985) concerning the distribution of the projected rotational velocities of Herbig Ae/Be stars: Herbig Ae/Be stars are depleted of slow rotators and rotate at intermediate velocities systematically more rapidly than $\mathrm{T}$ Tauri stars.

For F-G spectral types, a clear difference in $v \sin i$ between the PMS stars and their counterparts of luminosity class V can be deduced from Fig. 11. Concerning the B, A-types, there is a smooth transition in the PMS group from high $v \sin i$ values, typical of Be stars, to values lower than the average ones for objects of luminosity class V. This result fits nicely with the two scenarios proposed by Finkenzeller (1985) in which the further evolution of $v \sin i$ in PMS stars would depend on the relative strength of two opposite processes, namely, a speeding up due to a further contraction and the associated conservation of the angular momentum or a spin down due to a net angular momentum loss (e.g. by stellar winds). Depending of the leading mechanism, one could expect the formation of either a "normal" B, A-type main sequence star or a rapidly rotating $\mathrm{Be}$ star.

\section{Conclusions}

The spectral types of 70 stars and the projected rotational velocities, $v \sin i$ of 45 stars, mainly Vega-type and premain sequence (CTTs, ETTs and Herbig Ae/Be), have been determined. These are crucial parameters necessary for understanding the evolutionary sequence leading from the early stages of the pre-main sequence evolution to stars with disks and planets already formed. Future work, already in progress, includes the accurate determination of the stellar luminosities and metal abundances. The comparison of all data with those predicted from pre-main sequence evolutionary tracks will provide further insight into the physics of these stars. 
Acknowledgements. We are grateful to the referee, Prof. Gibor Basri, for his careful reading of the manuscript, suggestions and criticisms that helped us to improve the first version of this paper. We acknowledge the International Scientific Committee of the Canary Islands' Observatories for the allocation of observing time in the International Time programme. The work of C. Eiroa, B. Merín, B. Montesinos, A. Mora and J. Palacios has been supported in part by the Spanish grant ESP 98-1339. A. Mora wishes to thank Dr. J. R. Barnes for valuable discussions about echelle reduction procedures. Part of this research has made use of the SIMBAD database, operated at CDS, Strasbourg, France.

\section{References}

Abt, H. A., \& Morrel, N. I. 1995, ApJS, 99, 135

Allain, S., Fernández, M., Martín, E. L., \& Bouvier, J. 1996, A\&A, 314, 173

van den Ancker, M. E., De Winter, D., \& Tjin a Djie, H. R. E. 1998, A\&A, 330, 145

Aumann, H., Gillett, F., Beichman, C., et al. 1984, ApJ, 278, L23

Baade, D. 1989, A\&AS, 79, 423

Balachandran, S. C., Fekel, F. C., Henry, G. W., \& Uitenbroek, H. 2000, ApJ, 542, 978

Basri, G., \& Batalha, C. 1990, ApJ, 363, 654

Bernacca, P. L., \& Perinotto, M. 1970, in Contr. Oss. Astr. Univ. Padova, 239

Böhm, T., \& Catala, C. 1995, A\&A, 301, 155

Bouvier, J., Cabrit, S., Fernández, M., Martín, E. L., \& Matthews, J. M. 1993, A\&A, 272, 176

Castilho, B. V., Gregorio-Hetem, J., Spite, F., Spite, M., \& Barbuy, B. 1998, A\&AS, 127, 139

Catala, C., \& Bertout, C. 1990, in Evolution in Astrophysics, IUE Astron. in the Era of New Space Missions, ESA SP-310, 11

Cohen, M., \& Kuhi, L. V. 1979, ApJS, 41, 743

Corporon, P., \& Lagrange, A.-M. 1999, A\&AS, 136, 429

Díaz-Cordovés, J., \& Giménez, A. 1992, A\&A, 259, 227

Díaz-Cordovés, J., Claret, A., \& Giménez, A. 1995, A\&AS, 110,329

Drew, J. E., Busfield, G., Hoare, M. G., Murdoch, K. A., Nixon, C. A., \& Oudmaijer, R. D. 1997, MNRAS, 286, 538

Dudorov, A. E., \& Pudritz, R. E. 1994, in The Nature and Evolutionary Status of Herbig Ae/Be stars, ed. P. S. The, M. Pérez, \& E. P. J. van der Heuvel, ASP Conf. Ser., 62, 381

Dunkin, S. K., Barlow, M. J., \& Ryan, S. G. 1997, MNRAS, 286,604

Eiroa, C., Mora, A., Palacios, J., et al. (EXPORT) 2000, in Disks, Planetesimals and Planets, ed. F. Garzón, C. Eiroa, D. de Winter, \& T. Mahoney, ASP Conf. Ser., 219, 3

Eiroa, C., et al. (EXPORT), 2001, A\&A, 365, 110

Endal, A. S., \& Sofia, S. 1981, ApJ, 243, 625

Fekel, F. C. 1997, PASP, 109, 514

Fernández, M., \& Miranda, L. F. 1998, A\&A, 332, 629

Finkenzeller, U. 1985, A\&A, 151, 340

Folha, D. F. M., \& Emerson, J. P. 1999, A\&A, 352, 517

Fukuda, I. 1982, PASP, 94, 271

Ghosh, K. K., Apparao, K. M. V., \& Pukalenthi, S. 1999, A\&AS, 134, 359

Grady, C. A., Perez, M. R., Talavera, A., et al. 1996, A\&AS, 120,157
Grady, C. A., Sitko, M. L., Russel, R. W., et al. 2000, in Protostars and Planets IV, ed. V. Mannings, A. P. Boss, \& S. S. Russell (University of Arizona Press, Tucson), 613

Gray, D. F. 1992, in The observation and analysis of stellar photospheres (Cambridge University Press), 17

Guenther, E. W., \& Hessman, F. V. 1993, A\&A, 268, 192

Harris, A. W., Rauer, H., Cameron, A. C., et al. (EXPORT) 2000 in Disks, planetesimals and Planets, ed. F. Garzón, C. Eiroa, D. de Winter, \& T. Mahoney, ASP Conf. Ser., 219,454

Hessman, F. V., \& Guenther, E. W. 1997, A\&A, 321, 497

Howarth, I. D. 1983, MNRAS, 203, 301

Jaschek, C., \& Jaschek, M. 1990, The classification of stars (Cambridge University Press)

Jaschek, M., Jaschek, C., \& Andrillat, Y. 1988, A\&AS, 72, 505

Jasniewicz, G., Parthasarathy, M., De Laverny, P., \& Thevenin F. 1999, A\&A, 342, 831

Kurucz, R. L. 1993, CD-ROM 1-23, Smithsonian Astrophysical Observatory

Lecavelier des Étangs, A. 2000, in Disks, Planetesimals and Planets, ed. F. Garzón, C. Eiroa, D. de Winter, \& T. Mahoney, ASP Conf. Ser., 219, 308

Lecavelier des Étangs, A., Deleuil, M., Vidal-Madjar, A., et al. 1997, A\&A, 325, 228

Lindroos, K. P. 1986, A\&A, 156, 223

Magee, H. R. M., Dufton, P. L., Keenan, F. P., et al. 1998, A\&A, 338, 85

Mannings, V., Boss, A. P., Russell, S. S. (ed.), Protostars and Planets IV, Tucson University of Arizona Press

Mayor, M., \& Queloz, D. 1995, Nature, 378, 355

Millward, C. G., \& Walker, G. A. H. 1985, ApJS, 57, 63

Montes, D., Martín, E. L., Fernández-Figueroa, M. J., Cornide, M., \& de Castro, E. 1997, A\&AS, 123, 473

Moutou, C., Krelowski, J., D'Hendecourt, L., \& Jamroszczak, J. 1999, A\&A, 351, 680

Oudmaijer, R. D., et al. (EXPORT) 2001, A\&A, in press

Paunzen, E., Andrievsky, S. M., Chernyshova, I. V., et al. 1999, A\&A, 351, 981

Petrov, P. P., Zajtseva, G. V., Efimov, Y. S., et al. 1999, A\&A, 341,553

Ramella, M., Böhm, C., Gerbaldi, M., \& Faraggiana, R. 1989, A\&A, 209, 233

Ray, T. P., Sargent, A. I., Beckwith, S. V. W., et al. 1995, ApJ, 440, L89

Schmidt-Kaler, T. 1982, Landolt-Börnstein New Ser., vol. 2B, Chapt. Physical parameters of the stars (Springer-Verlag, New York)

Shajn, G., \& Struve, O. 1929, MNRAS, 89, 222

Smith, B. A., \& Terrile, R. J. 1984, Science, 226, 1421

Solano, E., \& Fernley, J. 1987, A\&AS, 122, 131

Stauffer, J. R., \& Hartmann, L. W. 1987, ApJ, 318, 337

Steele, I. A., Negueruela, I., \& Clark, J. S. 1999, A\&AS, 137, 147

Tonry, J., \& Davis, M. 1979, AJ, 84, 1511

Uesugi, A., \& Fukuda, I. 1982, in Revised Catalogue of Stellar Rotational Velocities, Depart. of Astronomy, Kyoto Univ., Japan

Valenti, J. A., Johns-Krull, C. M., \& Linsky, J. L. 2000, ApJS, 129,399

Vogel, S. N., \& Kuhi, L. V. 1981, ApJ, 245, 960

Welsh, B. Y., Craig, N., Crawford, I. A., \& Price, R. J. 1998, A\&A, 338, 674

Wolff, S. C., \& Simon, T. 1997, PASP, 109, 759 